\title{
Simplifying Sensemaking: Concept, Process, Strengths, Shortcomings, and Ways Forward for Information Systems in Contemporary Business Environments
}

\author{
Morteza Namvar \\ University of Wollongong \\ morteza.namvar@uow.edu.au \\ Jacob L. Cybulski \\ Deakin University

\section{Cynthia Su Chen Phang} \\ Swinburne University of Technology

\section{Yaw Seng Ee} \\ Swinburne University of Technology

\section{Kevin Tee Liang Tan} \\ Swinburne University of Technology
}

\section{Abstract}

Sensemaking is a popular and useful organizational behaviour concept that is gaining visibility in the field of information systems. However, it remains relatively unknown compared to more established information systems concepts like technology acceptance and resistance. To enhance and propel greater use of sensemaking in information systems, this article offers a systematic explanation of sensemaking, specifically focusing on its concept, process, strengths, and shortcomings, as well as discussing ways forward for information systems in contemporary business environments.

Keywords: Sensemaking; Decision making; Information systems; Concept; Process; Strengths; Limitations; Future research directions.

\section{Introduction}

Sensemaking, which was introduced and popularized by Karl Weick in 1969, is a concept that is accompanied by great irony. On the one hand, sensemaking aims to provide clarity and meaning to obscure phenomena (Weick, 1969, 1979, 1995); on the other hand, the conceptual boundaries (or process) of sensemaking have become obscure due to the mushrooming of sensemaking perspectives, leading to a state where "there is no single agreed definition of sensemaking" (Brown et al., 2015, p. 266).

Moreover, unlike other concepts in information systems-such as technology acceptance and resistance - that have been extensively reviewed to provide a snapshot of their progress in the field, sensemaking lacks a useful reference point that can offer scholars who are interested in the concept with such an overview.

To this end, the present paper aims to provide a systematic explanation of sensemaking, specifically focusing on its concept, process, strengths, and shortcomings, as well as ways forward for information systems in contemporary business environments. In doing so, this paper hopes to consolidate the essence of diverse sensemaking perspectives into a simplified blueprint for sensemaking. The aim is to stimulate greater usage of sensemaking to advance 
our understanding and application of information systems in contemporary business environments.

\section{Sensemaking}

Sensemaking exists due to the perception-expectation gap, which may occur in the form of breakdowns, surprises, discrepancies, or opportunities in organizational settings. However, to many entities, especially decision-makers, making sense of the data required to close this gap is often a challenging task. This is because data in its existential form is complicated, complex, and chaotic, and therefore, overwhelming. The following section on the concept of sensemaking deals with this challenge, and the subsequent sections introduce and discuss sensemaking in detail.

\subsection{The Concept}

Sensemaking has been defined in many ways by various scholars over time. For example:

- $\quad$ Russell et al. (1993, p. 269) considered sensemaking as "the process of searching for a representation and encoding data in that representation to answer task-specific questions,"

- Dervin (1998, p. 36) described sensemaking as "a methodology disciplining the cacophony of diversity and complexity without homogenizing it,"

- Weick et al. (2005, p. 409) suggested sensemaking as "the ongoing retrospective development of plausible images that rationalize what people are doing," and

- $\quad$ Brown et al. (2008, p. 1055) proposed sensemaking as "the processes of organizing using the technology of language-processes of labelling and categorizing for instance-to identify, regularize and routinize memories into plausible explanations."

To comprehensively put the essence of extant definitions of sensemaking into a straightforward perspective for future reference, this article considers the "what," "how," and "so what" questions relating to sensemaking. In doing so, it proposes a simple but holistic definition for sensemaking: Sensemaking is a process of clarifying and removing ambiguity and uncertainty by searching for and organizing similarities and differences from data sources through which goal-directed interpretations for decision-making are established. Therefore, sensemaking as a process is the foundation of knowledge creation, where the quality of sensemaking affects the quality of knowledge produced and the outcome of decisions predicated on that knowledge.

However, the peculiarities of this process are complex and varied. The process of sensemaking is scrutinized and discussed in the next section.

\subsection{The Process}

The sense that one initially and subsequently develops over time is predicated on a set of underpinnings, which has taken multiple forms over the years. For example:

- Dervin $(1983 ;$ 1998) contends that sensemaking as a methodology involves identifying and bridging three gaps that exist between situation and outcomenamely time, space, and movement. These gaps also interact with each other to create additional gaps-namely time-space, time-movement, and space-movement gaps. 
- $\quad$ Russell et al. (1993, p. 271) posit that sensemaking consists of three learning loopsnamely the generation loop (or the search for representations, where appropriate representations are created to capture important regularities in data sources-e.g. establish categories, comparison dimensions, specifications for clustering), the data coverage loop (or the instantiation of representations, where data of interest are identified and encoded into appropriate representations), and the representational shift loop (or the shift of representations, where sensemaking iterates between the generation and data coverage loops-e.g. to account for the discovery of residue, where original representations may be merged or split, for otherwise new representations may be created).

- Weick $(1995 ; 2008$, pp. 1404-1405) proposes that sensemaking is a project whose process is grounded on seven properties - namely identity construction (or the sense of self, as an individual [such as skills, knowledge, experience] or as an organization [such as structure, strategy, culture]), social context (or the associated meanings assigned to social support, consensual validation, and shared relevance in the actual, implied, or imagined presence of others-e.g. culture, education, and interaction among interdependent people shape the interpretation of events), retrospection (or the reflection on past events relevant to current interpretations [e.g. structured or unstructured internal and external data], which may be influenced by the extent of lapse time and recollection capabilities), salient cues (or the diagnosis and elaboration of traces for confirming evidence [e.g. alerts, metrics, patterns, key performance indicators]), ongoing projects (or the continuing or future events that can alter and shape current interpretations [e.g. data from business intelligence and analytics]), plausibility (or the adequacy of interpretation and associated justifications to proceed with current projects [e.g. plausible models]), and enactment (or the actions that one takes to inspect, interpret, or interact with observations-e.g. asking questions and inserting probes to make sense of a situation). Note that sensemaking is applicable to both business processes and projects.

- $\quad$ Pirolli and Card (2005) suggest that sensemaking consists of a set of sequential tasks - namely information development (or the gathering of information of interest), schema development (or the representation of gathered information in a schema that aids analysis), insight development (or the insights produced through the manipulation of representations), and product development (or the action or creation of a product predicated on the knowledge from sensemaking insights). The sequence of sensemaking tasks can follow two approaches: a bottom-up approach or a top-down approach. The former requires the sensemaker to search and filter, read and extract, schematize, build a case, and relate the information of interest (story tell), whereas the latter requires the sensemaker to search for support, evidence, relations, and information from the final product. Regardless of its approach, the quality of sensemaking is assured through two loops: the foraging loop (or the exploration-enrichment-exploitation cycle) and the sensemaking loop (or the problem structuring [i.e. the generation, exploration, and management of hypotheses], evidentiary reasoning [i.e. the marshalling of evidence to support or disconfirm hypotheses], and decision-making [i.e. the choosing of the best 
alternative for further action from the set of alternatives produced through the foraging loop]).

- $\quad$ Namvar et al. (2016) shed light on the notion of information systems in the form of business intelligence (e.g. standard reports, ad-hoc reports, query drilldowns, and alerts) and business analytics (e.g. statistical analysis, forecasting, predictive modelling, and optimization). The authors identify these as the key factors of consideration (or enablers) that sensemakers should take into account and use to improve the process (e.g. empower and maximize the returns) of sensemaking.

When an individualistic view is employed, the process of sensemaking proposed by each scholar appears distinct. However, when a collective view is taken, the crossroads between these underpinnings suggest that the different propositions by past scholars are, in fact, complementary to one another. Thus, similar to the consolidation of sensemaking definitions in the previous section, this article contends that an integration of underpinnings is necessary to address existing piecemeal understanding and to solidify future understanding of the sensemaking process (see Figure 1).

More specifically:

- The contention by Dervin $(1983,1998)$ sets the overarching tone to explain the motivation to engage in sensemaking - that is, to identify and bridge the gap - of time (past, present, future), space (context), movement (changes), or its combination (interaction) between an existing situation and an intended outcome through which sensemaking is applied as a knowledge-based solution-generation mechanism.

- $\quad$ The suggestion by Pirolli and Card (2005) lays the foundations for the sensemaking process in terms of its tasks (i.e. information, schema, insight, and product development), sequence (i.e. bottom-up or top-down), and quality-assurance loops (i.e. foraging and sensemaking loops).

- The proposition by Russel et al. (1993) in the form of learning loops (i.e. generation, data coverage, and representational shift loops) acts as sub-loops within the foraging loop (or as part of the exploration-enrichment-exploitation cycle).

- $\quad$ Finally, the sensemaking properties proposed by Weick $(1995,2008)$ and the business information systems suggested by Namvar et al. (2016) are positioned as factors to consider (or enablers) for the sequential tasks and quality-assurance loops in the sensemaking process. That is, data in the form of salient cues and ongoing projects serve as inputs to information development; identity construction, social context, retrospection, and plausibility serve as considerations that must be taken into account by the sensemaker as they affect the interpretations made during insight development; and the development of information received and the quality assurance loops are enacted with the support of business information systems in the form of business intelligence and business analytics.

\subsection{The Strengths}

The concept and process of sensemaking provided in this article have several upshots. First, sensemaking offers decision-makers with a structured process of dealing with ambiguity and uncertainty (Weick, 1995, 2008). Second, sensemaking offers explanations regarding the 
mental models of reality, their causes, and their use in reaching informed decisions with the support of existing and future information systems and technology (Boland, 2008; Namvar et al., 2016). Third, sensemaking links elements of the natural world, the social world, and the technology world, and thus is well-positioned to contribute to research inquiry in contemporary environments, such as in business information systems research (Gregor, 2006). Fourth, sensemaking offers the opportunity to address the challenges of using business information systems for decision-making (Hasan \& Gould, 2001), especially in modern datacentric organizations where business and information systems processes are intertwined (e.g. managerial decision-making and business intelligence and analytics) (Namvar \& Cybulski, 2014). In short, sensemaking, in a general sense, offers the opportunity to learn about, organize, and give meaning to obscure phenomena, and in a business sense, enables the transformation of complicated, complex, and chaotic organizational and marketplace data into the precipice of business acumen and realization among decision-makers, on behalf of organizations and in contemporary business environments.

\subsection{The Shortcomings}

When sensemaking was first introduced by Weick in 1969, information technology was limited to supporting core organizational processes, such as the management of human records, payroll processing, and producing financial reports. The notion of computer-based decision support was still in its infancy and modern communication technology was not available. It is not surprising that, over the years, sensemaking developed predominantly as a methodology for dealing with complex organizational phenomena (e.g. strategic change) (Gioia \& Chittipeddi, 1991; Maitlis \& Christianson, 2014) rather than as a practical information systemsbased tool for improving decision-making in organizations.

In spite of Weick's foresight in casting sensemaking as a data-intensive approach to organizational learning, the development of sensemaking missed out on opportunities offered by years of technological innovation. For instance, only in the last decade have we seen the advent and boom of new and inexpensive technologies, widely accessible to organizations and individuals, such as personal wireless networks (e.g. Wi-Fi and Bluetooth), mobile computing (e.g. smartphones), online media (e.g. YouTube) and social media (e.g. Twitter), on-demand online services (e.g. Uber), crowdsourcing (e.g. Mechanical Turk), cryptocurrencies (e.g. Bitcoin), and more. All these developments resulted in new socio-technological environments (e.g. online businesses), new types of business transactions (e.g. PayPal), new types of products (e.g. audio and video streams), new means of enacting business processes (e.g. Blockchain), new ways of sensing and collecting environmental cues (e.g. with remote sensors and meters), new ways of generating data (e.g. by leaving digital footprints via mobile phone), new kinds of business data or data that previously could not be processed (e.g. streams of unstructured text, audio, and video), fuzzy boundaries between an organization and an individual (e.g. work anywhere and anytime), new ways of creating personal and business identities (by creating instant impressions-e.g. Instagram), new ways of instant collaboration (e.g. via Skype or Trello), and more. Business circumstances are more uncertain than in the 1980s; change is proceeding at a higher pace and innovation has a steeper curve. In this world of technology-inspired change, the questions for sensemaking theorists are: How can we make sense of these new socio-organizational phenomena? How could sensemaking assist decisionmaking in a world disrupted by the introduction of new technologies? How could new technology improve sensemaking in support of decision-making? 
The consolidation of the concept and process of sensemaking outlined here, along with the ways forward for sensemaking presented in the next section, contributes some insights and paves the way for future research to provide more elaborate thoughts predicated on empirical research to respond to these questions.

\subsection{The Ways Forward}

Sensemaking results in individuals gaining an understanding of organizational complexity, which leads to desired action in the organization and its environment. The contemporary business environment is immersed in data and saturated with new business and personal technologies, some of which enhance while others disrupt decision-making processes. By relying on sensemaking processes, information systems-based decision-support tools-such as business intelligence and analytics - can be used and further improved to facilitate a more nuanced understanding of contemporary business environments, thus leading to more informed business decisions. This requires consideration of sensemaking properties when developing and using tools such as business intelligence and analytics, as follows:

- Retrospection: Traditional information systems and analytics tools are useful to provide a retrospective view of business environments by collecting, processing, and presenting structured data for decision-makers. However, new data analytics systems featuring text analytics and stream analytics tools can also process massive amounts of unstructured data, which is (often freely) available to business from internal or external sources.

- $\quad$ Salient cues: Many businesses today experience pressure from internal and environmental change, which confounds the collection of data cues and potentially leads to inferior data quality and decision-making uncertainty. Through data modelling, aggregation, and clustering, business intelligence and analytics tools can assist in rapidly reducing the volume, veracity, and volatility of data to a small number of relevant cues, such as alerts, key performance indicators, metrics, and patterns, among others, which can then be used to assign meaning to equivocal business situations and establish triggers for action.

- Plausibility: Due to the quality and volatility of data collected from open data sources, decisions based on such sources are subject to considerable uncertainty and risk. Plausible models developed with predictive analytics methods can, however, provide decision-makers with measures to weigh in various risk factors and with opportunities to apply their own expertise and domain knowledge, especially in situations where all the relevant factors and parameters cannot be incorporated into predictive models.

- Identity construction (individual) and social context: Decision-makers can also rely more extensively on the knowledge and experience of their team members, who can be accessed via collaborative (e.g. Microsoft Teams) and business social media (e.g. Yammer) facilities for sharing and reviewing online reports. Many such reports can be delivered in actionable form on portable devices. Thus, they readily fit the work patterns of a new generation of decision-makers who work anywhere and anytime. Tough decisions and hard cases (handling which may exceed inhouse skills), could be referred to the wisdom of crowds in the form of questions, problems, and competitions (e.g. Quora and Kaggle). 
- Identity construction (organization), ongoing projects, and enactment: Notwithstanding the advances in and proliferation of technology in recent times, one of the major limitations of current technologies is the lack of any direct link between decisionsupport tools and enactment processes, which is an important element in organizational sensemaking. The enactment of a decision-maker's own vision of the organizational process is still elusive; possible solutions include involving decision-makers in report generation and model building via self-service workflow analytics with interactive user-friendly drag-and-drop interfaces (e.g. Microsoft Power BI). The main requirement of the latter activity would be to alter the organizational identity (or capacity) in using business intelligence and analytics, as reflected in the structure, strategy, culture, and processes (whether formalized or enacted), all of which support internal collaborative activities, external presence, and overall behaviour. If resistance is present, potential behavioural remedies through priming measures may be considered (Lim, 2015).

\section{Conclusion}

In the increasingly competitive business landscape of today, organizations rely on massive amounts of data to monitor and analyse changes in their business environment. Business information systems, such as business intelligence and analytics, assist in this process by turning data into evidence that is capable of supporting decision-making. With rapid organizational and technological changes, and given the growing amount of shared data and data availability, it goes without saying that business decisions must also rest on a sound understanding of this complexity, for which sensemaking is crucial. Specifically, this article has made clear that sensemaking as an approach is suitable for continuously gaining an understanding of contemporary business environments and providing additional insights into decision-making processes. Sensemaking provides a useful theoretical lens for studying factors that influence organizations in their move toward informed decision-making. This article provides a consolidated overview of sensemaking in the form of its concept, process, strengths, and shortcomings, as well as ways forward for information systems in contemporary business environments, with a special focus on new ways of developing and using existing and future business intelligence and analytics more effectively.

\section{Acknowledgement}

Many thanks to Weng Marc Lim, the editor, and the anonymous reviewers for their constructive feedback to improve the quality of the paper. 


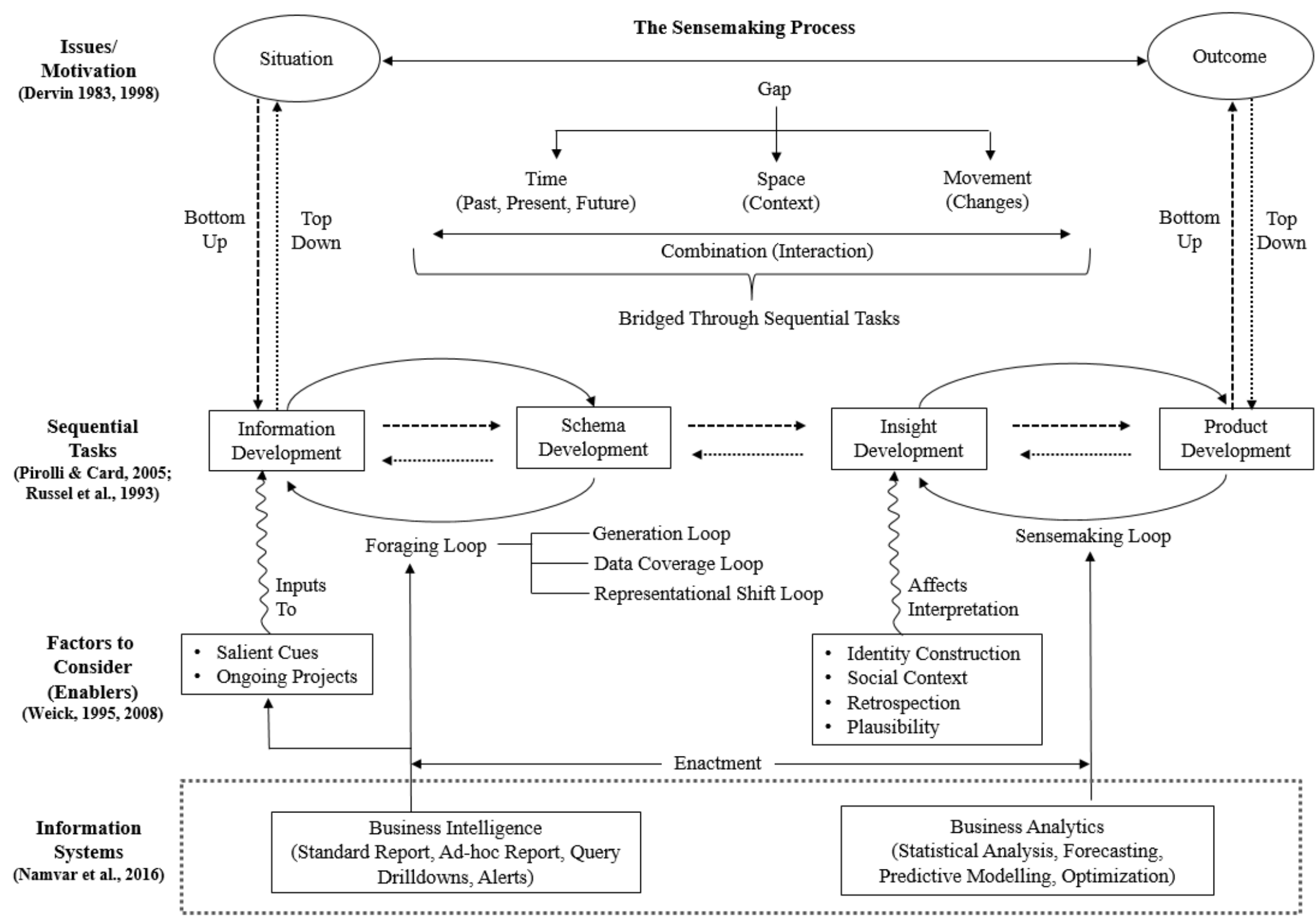

Figure 1

The Sensemaking Process 


\section{References}

Boland, R. J. (2008). Decision Making and Sensemaking. In Handbook on Decision Support Systems 1 (pp. 55-63). New York: Springer. doi: https://doi.org/10.1007/978-3-540-48713-5_3

Brown, A. D., Stacey, P., \& Nandhakumar, J. (2008). Making Sense of Sensemaking Narratives. Human Relations, 61(8), 1035-1062. doi: https://doi.org/10.1177/0018726708094858

Brown, A. D., Colville, I., \& Pye, A. (2015). Making sense of sensemaking in organization studies. Organization Studies, 36(2), 265-277. doi: https://doi.org/10.1177/0170840614559259

Dervin, B. (1998). Sense-Making Theory and Practice: An Overview of User Interests In Knowledge Seeking and Use. Journal of Knowledge Management, 2(2), 36-46. doi: https://doi.org/10.1108/13673279810249369

Gioia, D. A., \& Chittipeddi, K. (1991). Sensemaking and Sensegiving in Strategic Change Initiation. Strategic Management Journal, 12, 433-448. doi: https://doi.org/10.1002/smj.4250120604

Gregor, S. (2006). The Nature of Theory in Information Systems. MIS Quarterly, 611-642. doi: https://doi.org/10.2307/25148742

Hasan, H., \& Gould, E. (2001). Support for the Sense-Making Activity of Managers. Decision Support Systems, 31(1), 71-86. doi: https://doi.org/10.1016/S0167-9236(00)00120-2

Lim, W. M. (2015). Enriching Information Science Research through Chronic Disposition and Situational Priming: A Short Note for Future Research. Journal of Information Science, 41(3), 399-402. doi: https://doi.org/10.1177/0165551515577913

Maitlis, S., \& Christianson, M. (2014). Sensemaking in Organizations: Taking Stock and Moving Forward. Academy of Management Annals, 8(1), 57-125. doi: https://doi.org/10.1080/19416520.2014.873177

Namvar, M., \& Cybulski, J. (2014). BI-based Organizations: A Sensemaking Perspective. Proceedings of the International Conference on Information Systems 2014 (ICIS 2014), Auckland, New Zealand.

Namvar, M., Cybulski, J., \& Perera, L. (2016). Using Business Intelligence to Support the Process of Organizational Sensemaking. Communications of the Association for Information Systems, 38, 330-352. doi: https://doi.org/10.17705/1CAIS.03820

Pirolli, P., \& Card, S. (2005). The Sensemaking Process and Leverage Points for Analyst Technology As Identified Through Cognitive Task Analysis. Proceedings of International Conference on Intelligence Analysis (Vol. 5).

Russell, D. M., Stefik, M. J., Pirolli, P., \& Card, S. K. (1993). THE Cost Structure of Sensemaking (pp. 269-276). Proceedings of the INTERACT'93 and CHI'93 Conference On Human Factors In Computing Systems, ACM. doi: https://doi.org/10.1145/169059.169209

Weick, K. E. (2008). Sensemaking. In Clegg, S. R., \& Bailey, J. R., International Encyclopaedia of Organization Studies (pp. 1404-1406). Thousand Oaks, CA: Sage Publications.

Weick, K. E. (1995). Sensemaking in Organizations. Thousand Oaks, CA: Sage Publications.

Weick, K. E. (1969). The Social Psychology of Organizing. Reading, MA: Addison-Wesley. 
Weick, K. E. (1979). The Social Psychology of Organizing (2nd Ed.). New York: McGraw-Hill.

Weick, K. E., Sutcliffe, K. M., \& Obstfeld, D. (2005). Organizing and the Process of Sensemaking. Organization Science, 16(4), 409-421. doi: https://doi.org/10.1287/orsc.1050.0133

Copyright: (C) 2018 Namvar, Cybulski, Phang, Ee \& Tan. This is an open-access article distributed under the terms of the Creative Commons Attribution-NonCommercial 3.0 Australia License, which permits non-commercial use, distribution, and reproduction in any medium, provided the original author and AJIS are credited.

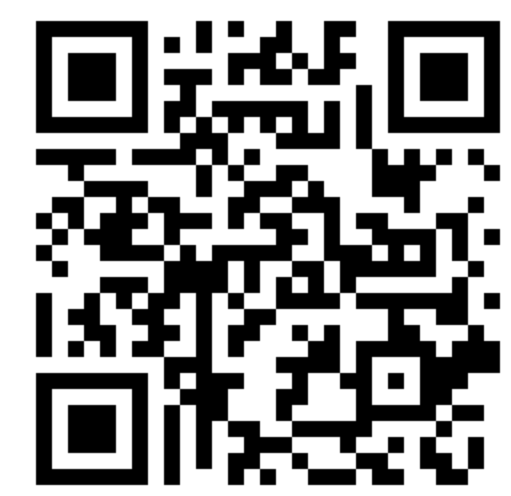

